\title{
Looking to the future
}

In 2012, we were starting to face the crisis in acute medical care. An RCP report published that year, Hospitals on the edge? The time for action, ${ }^{1}$ painted a stark picture: there had been a $37 \%$ rise in patient admissions over 10 years, and these patients were older and with more complex comorbidities. Physicians and their teams struggled to cope with the rising tide. The result was not always centred around the care of the patient, or even safe. Continuity of care was fragmented, with patients being moved multiple times and handover procedures poor; mortality was on average $10 \%$ higher in patients admitted over the weekends. A workforce crisis loomed, with trainees becoming less willing to follow the increasingly pressured career path of the medical registrar in general internal medicine. The transfer of patients back into the community was often creaking.

In response, I appointed the Future Hospital Commission (FHC) to review all aspects of the design and delivery of inpatient hospital care. The Commission was formally independent of the RCP, under the chairmanship of Sir Michael Rawlins and led by Professor Tim Evans, and brought together panels of experts from medical and other disciplines, together with patient representatives, to build a vision of comprehensive medical care in the hospital of the future. The FHC also scoured the UK for examples of successful practice from across a range of trusts. This broad base of input into the Commission proved to be essential to its success; the outcome, set out in the report Future hospital: caring for medical patients, ${ }^{2}$ was a bold and far-reaching vision for a new model of clinical care for acute medical patients, together with an examination of the changes to systems and culture that would be necessary to achieve this.

However, this publication of the FHC's recommendations was only the beginning. The College has since set up the Future Hospital Programme, which will now develop and implement the FHC's vision for the future of medical care across hospital and community settings, with a focus on driving real change in the way services are delivered and structured in hospitals and the local community. One important strand will be the development of a partnership programme, in which partner hospitals and healthcare services will pilot, develop and implement the Future Hospital Commission's recommendations.

The RCP early realised the need to provide a sustainable, long-term forum for the output from these pilots, together with other evidence-based research, debate and analysis of evidence around the principles of the Future Hospital, including lessons from abroad. There are clear advantages of the established journal model of publishing, with its well-tested systems for peer review and dissemination of information, and I am delighted that you now have the first issue of the Future Hospital Journal in your hands, or on your screen.
The RCP's existing journal, Clinical Medicinerelaunched in 2000, phoenix-like, out of the Journal of the Royal College of Physicians of London, first published in 1966 - has long been where the practising physician can find content that is of use clinically and professionally. Its younger sister will also be of relevance to practising physicians eager to lead and improve services in their local hospitals, but we hope that it will also have a wider remit, targeting as well healthcare managers, healthcare policymakers and academic researchers in healthcare services. There are many journals publishing on health systems, but the Future Hospital Journal will be the first to have a particular focus on secondary medical care and the system and culture changes that are needed in this setting.

The Future Hospital Journal is looking to the future, but looking back to a time when the exchange of ideas and knowledge in scholarly journals was gaining traction across Europe, to the Transactions of the Royal College of Physicians, the RCP's first journal, which preceded the Journal of the Royal College of Physicians and was launched in 1772. The editors set out their aspirations:

This advancement [in natural knowledge] hath been greatly owing to those learned societies in Europe who, by collecting papers relating to this branch of knowledge, have preserved many useful observations, which would otherwise have been lost.

As the art of physic hath been much improved by this method of communicating observations, the College of Physicians in London are desirous of furthering a design so worthy of their attention; and are ready to receive any medical paper that shall be presented to them, in order to publish the most useful.

My hope today in 2014 is that the Future Hospital Journal will likewise collect 'many useful observations' on service design from hospitals and their communities around the UK and the world. Our success will be dependent on the willingness of doctors, researchers and others working in the health service to submit articles sharing the outcomes of local innovation - whether positive or, just as important, negative. Through a robust system of peer review, the RCP, represented by Tim Evans and the rest of the editorial board, will select 'the most useful' and disseminate them to readers who are in a position to implement the best innovative practice in their own trusts.

As this first issue of the Future Hospital Journal appears, the end of my presidency will be near. The Future Hospital Commission has been an important part of my tenure, much as I regret the situation that mandated it. I have every hope that the Future Hospital Journal will flourish - enhancing communication, stimulating discussion and debate, and sharing successful innovation across the NHS. 
1 Royal College of Physicians. Hospitals on the edge? The time for action. London: RCP, 2012. Available online at www.rcplondon.ac. uk/sites/default/files/documents/hospitals-on-the-edge-report.pdf.

2 Future Hospital Commission. Future hospital: caring for medical patients. A report from the Future Hospital Commission to the Royal College of Physicians. London: Royal College of Physicians, 2013. Available online at www.rcplondon.ac.uk/sites/default/files/futurehospital-commission-report.pdf.

Sir Richard Thompson

\section{Access Future Hospital Journal online}

The Future Hospital Journal is hosted online on the industry-leading HighWire platform.

Functionality to help you get the most from the journal content includes:

> Pop-up abstracts and a homepage column alongside all content, enabling quick and easy browsing and navigation

$>$ Reference lists with onward linking, including free access to all HighWire-hosted articles

$>$ Enhanced search functionality to help you find the content you need
> Tailored alerts so you never miss the content most useful to you

$>$ Features such as 'Download directly to PowerPoint' and 'Email to a friend' that make it easy to share content with colleagues $>$ Publication ahead of print for accepted articles

HighWire, which is affliated to Stanford University, works closely with publishers on a continuous programme of technology improvements, giving RCP journals a future-proof platform for its current content and back archive.

Access the Future Hospital Journal online today at: www.futurehosp.rcpjournal.org 